\title{
3D post-processing technique highlights lesions: A thought triggered by an elderly, severe, complicated COVID-19 patient
}

\author{
Lei Tang ${ }^{1, *}$, Xiaoyong Zhang ${ }^{1, *}$, Yuquan Wang ${ }^{1}$, Rongpin Wang ${ }^{1}$, Xianchun Zeng ${ }^{1,2, \#}$ \\ ${ }^{1}$ Department of Radiology, Guizhou Provincial People's Hospital, Key Laboratory of intelligent medical imaging analysis and \\ accurate diagnosis of Guizhou Province, International Exemplary Cooperation Base of Precision Imaging for Diagnosis and \\ Treatment, Guiyang, Guizhou 550002, China \\ 2 Jiangjunshan Hospital of Guizhou Province, Guiyang, Guizhou 550002, China
}

\author{
*These authors contributed equally to this work. \\ \# Correspondence: Xianchun Zeng \\ E-mail: zengxianchun04@foxmail.com \\ Received: June 17, 2020 \\ Published: August 11, 2020
}

\begin{abstract}
The coronavirus disease 2019 (COVID-19) has swept across all countries in the world within half a year, which is taking the world by surprise. We reported an elderly, severe, complicated COVID-19 case, describing the clinical manifestations, laboratory examinations, CT findings, diagnosis, treatment and dynamic disease course. 3D post-processing technique was used to reconstruct different pulmonary lesions, and it was found to be useful for highlighting and observing them, thereby helping diagnose and distinguish different diseases.

Keywords: coronavirus disease 2019 (COVID-19), CT imaging, 3D post-processing

Abbreviations: COVID-19, Coronavirus Disease 2019; PHEIC, Public Health Emergency of International Concern; WHO, World Health Organization; RT-PCR, real-time reverse transcription polymerase chain reaction.

To cite this article: Lei Tang, et al. 3D post-processing technique highlights lesions: A thought triggered by an elderly, severe, complicated COVID-19 patient. Inflamm Cell Signal 2020; 7: e1170. doi: 10.14800/ics.1170.
\end{abstract}

\section{Introduction}

The coronavirus disease 2019 (COVID-19) has been declared as a Public Health Emergency of International Concern (PHEIC) by World Health Organization (WHO) $)^{[1-4]}$. The elderly and patients with chronic underlying diseases tend to have severe conditions and poor prognosis ${ }^{[5]}$. We report an elderly, severe, complicated COVID-19 case, especially focusing on $\mathrm{CT}$ features and the role of $3 \mathrm{D}$ post-processing technology in diagnosis.

\section{Case Presentation}

A 85-year-old woman who visited Wuhan 14 days ago developed cough, phlegm, and loss of appetite on February 5, 2020. By her own account, she had no special medical history. Three days later, she was admitted to hospital and received laboratory tests, which showed normal white blood cell count and lymphocyte count, elevated C-reactive protein (16.06 $\mathrm{mg} / \mathrm{L}$; normal range: $0-5 \mathrm{mg} / \mathrm{L})$, increased erythrocyte sedimentation rate $(54 \mathrm{~mm} / \mathrm{h}$; normal range: $2-20.9 \mathrm{~mm} / \mathrm{h})$ and rised D-dimer level $(8.63 \mu \mathrm{g} / \mathrm{ml}$; normal range: $0-1.5 \mu \mathrm{g} / \mathrm{ml})$.

Plain chest CT revealed multiple patchy ground glass opacities in bilateral lungs, and three masses in the apical segment of right lung and the inferior lobe of both lungs (Figure 1). 3D post-processing images (CT post-processing 


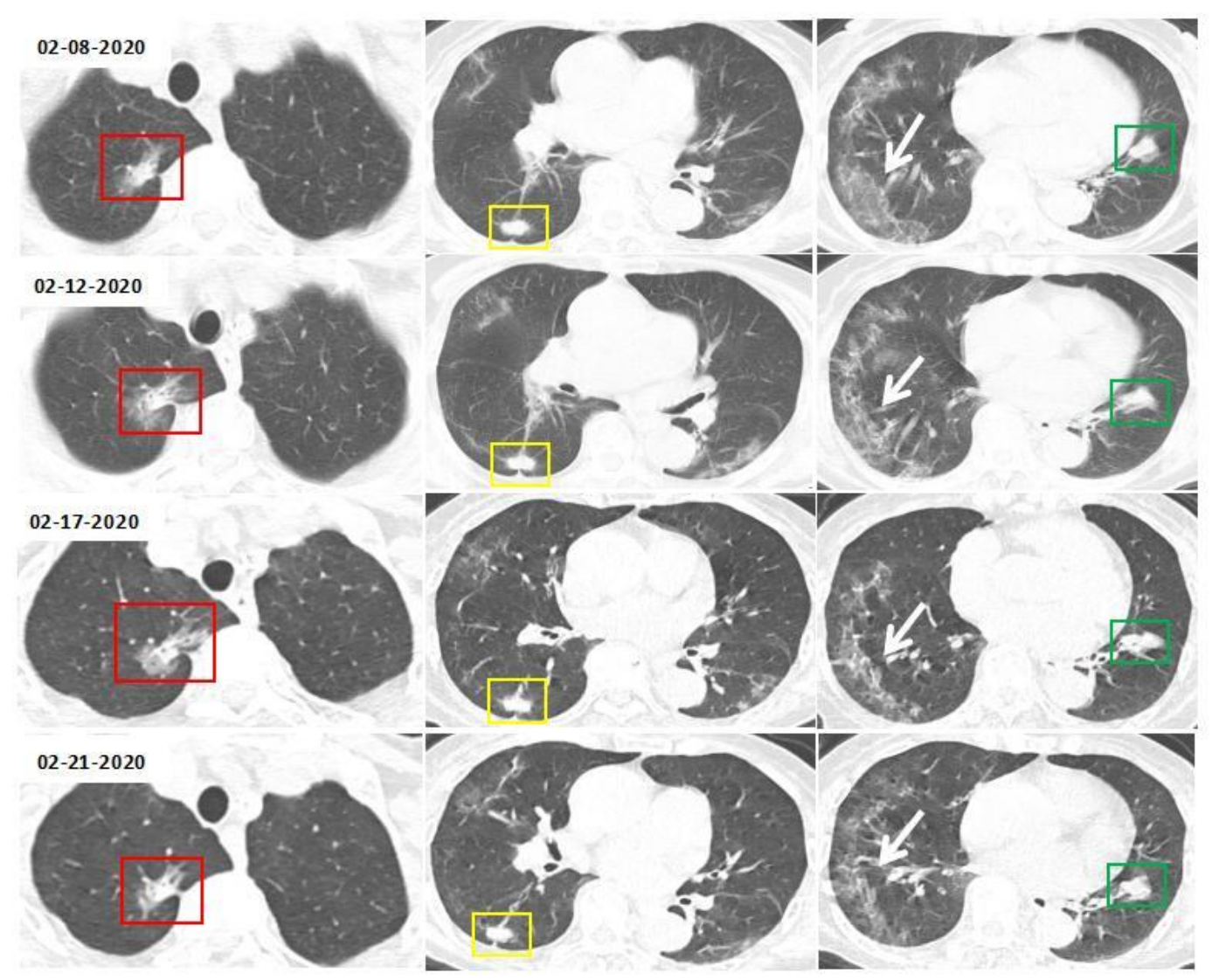

Figure 1. CT images in an elderly, severe, complicated COVID-19 patient. Transverse images reveal the dynamic changes of patchy peripheral ground glass opacities (white arrow) and three blocky shadows in the apical segment of right lung and the inferior lobe of both lungs. The exudation around the lesion in the apical of right upper lobe shows a tendency of increasing gradually and then absorption (red frame). The two mass shadows in the posterior segment of lower lobe of right lung (yellow frame) and the anteromedial basal segment of inferior lobe of left lung (green frame) present no significant dynamic changes.

software, Syngo.via, Version VB20, Siemens Healthcare, Germany) clearly highlighted different foci in both lungs, including location, morphology, distribution, surrounding invasion, etc (Figure 2 and 3). The two blocky shadows in the apical segment of right upper lobe and the anteromedial basal segment of inferior lobe of left lung were considered to be chronic inflammatory lesions according to morphological characteristics. Although the mass in the posterior segment of lower lobe of right lung with lobulated shape, spicules of margin, and pleural indentation sign, may be malignant, she was too old and weak to undergo further needle biopsy or surgery to determine the nature of the lesion (Figure 2). A pharyngeal swab was positive for novel coronavirus on realtime fluorescence polymerase chain reaction (RT-PCR) test, and she was diagnosed with severe COVID-19 due to low oxygen saturation (SPO2 <93\%). Then she was isolated and given oxygen inhalation, moxifloxacin, and abedore treatment.

After receiving 4 days of treatment, the patient's symptoms were relieved, but CT reexamination suggested progress, with enlarged peripheral patchy ground glass opacities and increased exudation around the lesion in the apical of right upper lobe (Figure 1). For the treatment, lopinavir/ritonavir and interferon were added. In the following 10 days, her Ddimer level continued to rise, fluctuating at $22.88-48.14 \mu \mathrm{g} / \mathrm{ml}$. Pulmonary vascular CTA showed no pulmonary embolism, lower limb vascular doppler ultrasound showed no venous thrombosis, and the patient had no no special discomfort. So clinicians considered the elevation of D-dimer to be associated with COVID-19 infection. However, during this period, two follow-up CT suggested reduced ground glass opacities, and three RT-PCR reexaminations were negative. On February 25, 2020, the patient was transferred to the local COVID-19 designated hospital for further treatment.

\section{Discussion}

Unenhanced CT examination is one of the major clinical diagnostic criteria for COVID-19 and plays an important role in early diagnosis, disease assessment and follow-up, because 

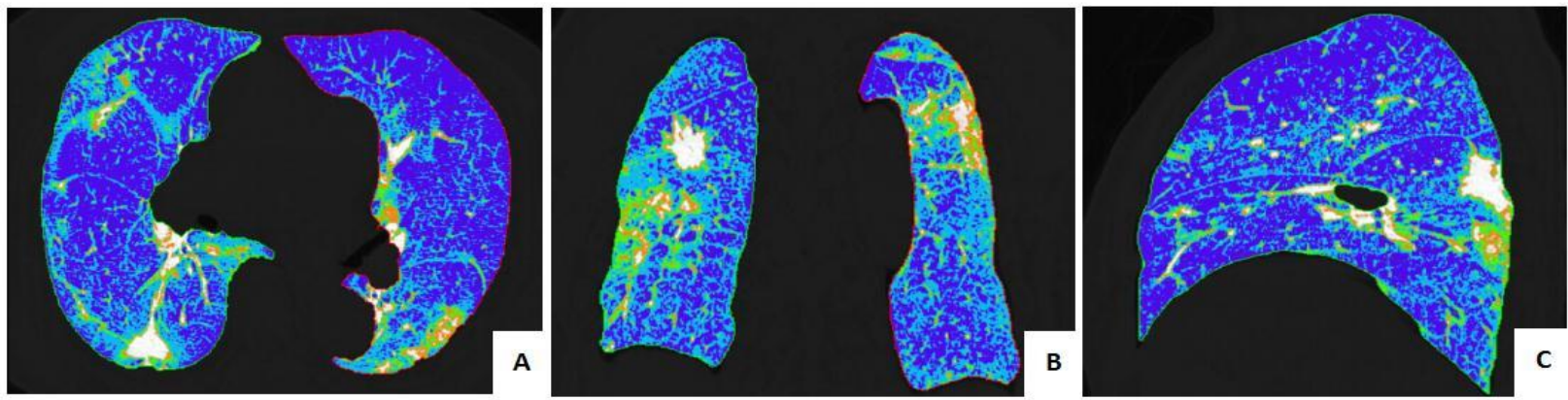

Figure 2. CT images in an elderly, severe, complicated COVID-19 patient. A-C, Pseudocolor images of central layer of lesion highlight the morphological characteristics of the mass and its relationship with the adjacent pleura. The mass in the posterior segment of lower lobe of right lung has lobulated shape, short thorn and vessel convergence, involving the posterior pleura and oblique fissure.
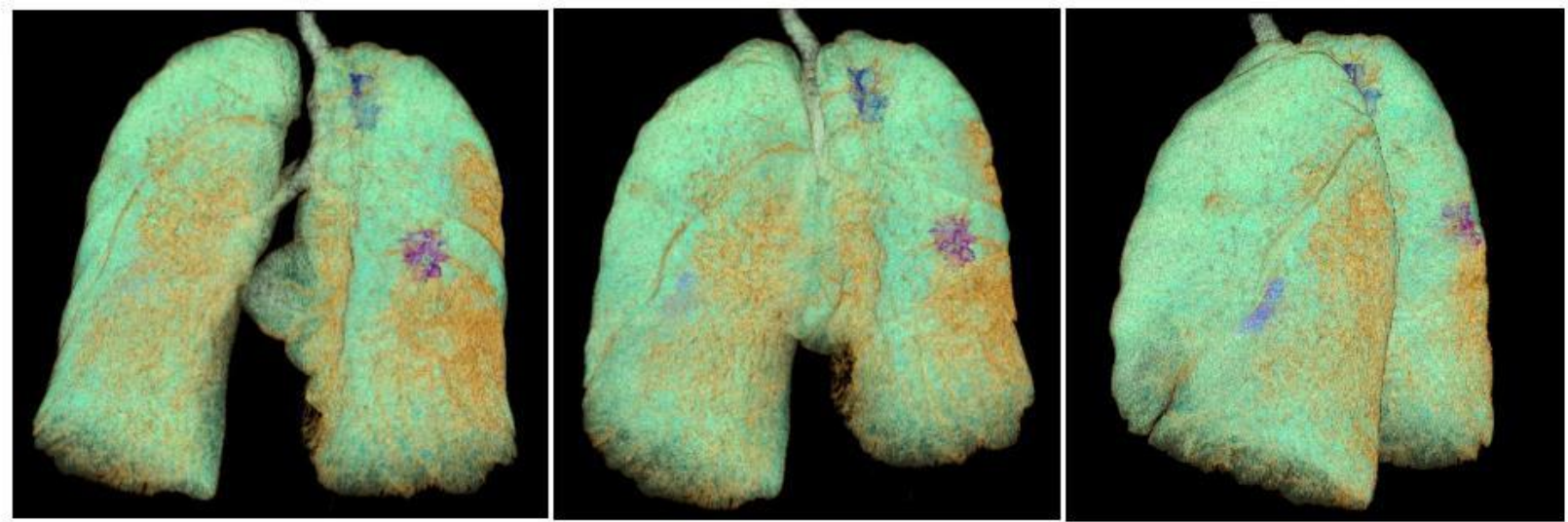

Figure 3. 3D post-processing CT images in an elderly, severe, complicated COVID-19 patient. 3D volume reconstruction and area growth technique simultaneously reveal normal lung tissue, pneumonic exudation and three mass shadows, not only helping to assess the extent and distribution of COVID-19 infection, but also highlighting the location and morphology of the three lumpy opacities.

CT findings can indicate the progression of the disease $\mathrm{e}^{[6]}$. However, there were two contradictions between clinic and CT imaging in the course of disease in the patient we reported. While her clinical symptoms were in remission, the CT scan showed more ground glass shadows and exudation. While her D-dimer level continued to rise (suggesting a worsening of the infection), follow-up CT revealed gradual absorption of lesions. Therefore, for the elderly patients and patients with chronic diseases, not only the disease is more serious and prognosis is worse, but also clinical manifestations and CT imaging may be inconsistent. For the elderly, severe and complicated patients, CT examination should not be solely relied on, but should be combined with clinical and laboratory examinations to further evaluate the disease changes.

As demonstrated by Liu T, et al and Peng Liu, et al, sophisticated reconstruction algorithms could provide better visualization of pulmonary lesions in COVID-19 patients with radiologists ${ }^{[7,8]}$. The lung foci in the patient we reported were multiple and complex, but 3D post-processing technology clearly showed the distribution, morphological characteristics and surrounding invasion of the lesions. Taking the mass in the posterior segment of lower lobe of right lung as an example, the pseudocolor images of central layer of lesion highlighted the morphological characteristics of the mass and its relationship with the adjacent pleura. Lobulated shape, short thorn, vessel convergence, and the involvement of the posterior pleura and oblique fissure, highly suggested a possibility of malignancy. Further 3D volume reconstruction and area growth technique visually marked the position and shape of the three blocky shadows, which were clearly distinguished from the surrounding patchy ground glass opacities. Therefore, 3D post-processing techniques can help to display the distribution and characteristics of different lesions in complicated COVID-19 cases, thereby helping diagnose and differentiate the diseases. 


\section{Ethics Approval}

This study was approved by the ethics committee of Guizhou Provincial People's Hospital.

\section{Patient Consent for Publication}

A written informed consent was obtained from the patient for publication of the information about her that appears within this case report.

\section{Conflicting Interests}

The authors declare that they have no conflict of interests.

\section{Acknowledgments}

This study was supported by Guizhou Science and Technology Project (QKHZC[2020]4Y002), Guiyang Science and Technology Project (ZKXM[2020]41), and Guizhou Science and Technology Department Key Lab Project (QKF[2017]25).

\section{References}

1. Wu F, Zhao S, Yu B, et al. A new coronavirus associated with human respiratory disease in China. Nature. 2020. [doi:10.1038/ s41586-020-2008-3]
2. World Health Organization. Novel Coronavirus (2019-nCoV) situation reports. Accessed 11 February 2020, https://www.who. int/emergencies/diseases/novel-coronavirus-2019/situation-reports.

3. Hu X, Chen J, Jiang X, et al. CT imaging of two cases of one family cluster 2019 novel coronavirus (2019-nCoV) pneumonia: inconsistency between clinical symptoms amelioration and imaging sign progression. Quant Imaging Med Surg. 2020; 10:508-510.

4. Li Q, Guan X, Wu P, et al. Early Transmission Dynamics in Wuhan, China, of Novel Coronavirus-Infected Pneumonia. N Engl J Med. 2020. [doi: 10.1056/NEJMoa2001316 (2020)].

5. National Health Commission of the People's Republic China: The diagnosis and treatment guidelines of COVID-19 (6th edition). Accessed 7 March 2020, http://www.nhc.gov.cn/yzygj/s7653p/ 202002/3b09b894ac9b4204a79db5b8912d4440.shtml.

6. Pan F, Ye T, Sun P, et al. Time Course of Lung Changes On Chest CT During Recovery From 2019 Novel Coronavirus (COVID-19) Pneumonia. Radiology. 2020; 200370-200370.

7. Liu T, Huang P, Liu H, et al. Spectrum of Chest CT Findings in a Familial Cluster of COVID-19 Infection. Radiology: Cardiothoracic Imaging. 2020; 2: e200025.

8. 8. Peng Liu, Xian-zhen Tan. 2019 Novel Coronavirus (2019-nCoV) Pneumonia. Radiology. 2020; 295:19. 